\title{
FINE STRUCTURE OF THE INTEGRAL EXPONENTIAL FUNCTIONS BELOW $2^{2^{x}}$
}

\author{
BERND I. DAHN
}

\begin{abstract}
Integral exponential functions are the members of the least class of real functions containing 1 , the identity function, and closed under addition, multiplication, and binary exponentiation sending $f$ and $g$ to $f^{g}$. This class is known to be wellordered by the relation of eventual dominance. It is shown that for each natural number $n$ the order type of the integral exponential functions below $2^{x^{n}}$ (below $x^{x^{n}}$ ) is exactly $\omega^{\omega^{2 n-1}}\left(\omega^{\omega^{2 n}}\right.$ respectively). The proof, using iterated asymptotic expansions, contains also a new proof that integral exponential functions below $2^{2^{x}}$ are wellordered.
\end{abstract}

In 1956 Skolem [Sk] investigated the class of integral exponential functions--the least class containing 1 , the identity function, and closed under additon, multiplication and binary exponentiation sending $f, g$ to $f^{g}$. This class of functions, denoted by Sk, is linearly ordered by the relation $<$ of eventual dominance (see $[\mathbf{H}]$ ). Skolem asked whether this is a wellordering and-if so--what is its order type. he pointed out a subset of Sk having the order type $\varepsilon_{0}=\sup \left\{\omega, \omega^{\omega}, \omega^{\omega}, \ldots\right\}$. Ehrenfeucht $[\mathbf{E}]$ proved in 1973 that the eventual dominance relation is in fact a wellordering. He applied a powerful graph theoretical result due to Kruskal.

Recently van den Dries and Levitz [DL] showed that the integral exponential functions below $2^{2^{x}}$ are wellordered of type $\omega^{\omega^{\omega}}$. Putting for $f \in \operatorname{Sk} \operatorname{Sk}(f):=\{g \in$ Sk: $g<f\}$, their proof gives also that

$$
\omega^{\omega^{2 n-1}} \leq\left|\operatorname{Sk}\left(2^{x^{n}}\right)\right|<\omega^{\omega^{n^{2}}}
$$

for each $n \in \omega$. It is the aim of this paper to show that in fact

$$
\omega^{\omega^{2 n-1}}=\left|\operatorname{Sk}\left(2^{x^{n}}\right)\right| \text {. }
$$

It is worth noting that we shall not refer to Ehrenfeucht's paper $[\mathbf{E}]$ anymore, i.e., we will also obtain a new proof that $\operatorname{Sk}\left(2^{2^{x}}\right)$ is wellordered.

The methods of the present paper were inspired by [DL] and came up in numerous discussions with Peter Göring, whom the author thanks very much for his patience and active interest.

For the reader it will be helpful to recall from [D] and [DG] that exponentiallogarithmic terms, especially integral exponential functions, can be represented in a canonic way by iterated powerseries of length $\omega$ or, optionally, as powerseries of transfinite length.

Let us first state some conventions and notations. If $K, L$ are ordered fields, $K \subseteq L, a, b \in L$, then we write $a \ll b \bmod K$ iff $b \neq 0$ and $|a / b|<\varepsilon$ for each

Received by the editors September 23, 1985.

1980 Mathematics Subject Classification (1985 Revision). Primary 03D20, 26A12; Secondary $06 \mathrm{~F} 05$. 
positive $\varepsilon \in K, a \sim b \bmod K$ means $a-b \ll a \bmod K$ or equivalently $-a, b \neq 0$ and $(a / b)-1 \ll 1 \bmod K$. We write $o_{K}(a)$ for $b \in L$ such that $b \ll a \bmod K$.

If $A$ and $B$ are sets and $f$ is a function defined on $A \times B$, then we put $f(A, B)=$ $\{f(a, b): a \in A, b \in B\}$. Similarly, if $r$ is a relation then $r(A, B)$ abbreviates " $r(a, b)$ for all $a \in A, b \in B$ ". For $a \in A f(a, B), r(a, B)$ replace $f(\{a\}, B), r(\{a\}, B)$ respectively. $R$ denotes the set of all positive real numbers. If $A$ is a subset of an additive group, then $A^{+}$denotes the set of all finite sums of elements of $A$.

The proof of the following lemma is adopted from $[\mathbf{F}]$.

LEMMA 1. Suppose $A$ is a wellordered subset of a linearly ordered additive abelian group and $A>0$. Then $A^{+}$is wellordered.

PROOF. Assume $a$ is the least element of $A$ such that there is a natural number $n$ such that $A^{+}$contains an infinite descending chain below $n a$. Let $n_{0}$ be minimal with this property and let $s_{1}>s_{2}>\cdots$ be such a chain. For each $s_{i}$ we choose a fixed representation of $s_{i}$ as a sum of elements of $A$ and let $a_{i}$ denote the maximal summand of this representation. Then $a_{i} \geq a$ by the choice of $a$ since $s_{i+1}<s_{i}<$ $n a_{i}$ for some $n$. Without loss of generality we can also assume $a_{i} \leq a_{i+1}$ for each $i$. This yields $s_{i+1}-a_{i+1}<s_{i}-a_{i} \leq s_{i}-a \leq\left(n_{0}-1\right) a$, contradicting the choice of $n_{0}$.

LEMMA 2. Suppose $A$ is a wellordered subset of a linearly ordered additive abelian group and $A>0$. Then each element of $A^{+}$has only finitely many representations as a sum of elements of $A$.

Proof. Suppose $a^{*}$ is the least element of $A^{+}$having infinitely many representations as a sum of elements of $A$. Since for each such representation $a^{*}=$ $a_{0}+a_{1}+\cdots+a_{n}$ we have $a_{1}+\cdots+a_{n}<a^{*}$, each $a_{0} \in A$ can occur only in finitely many representations of $a^{*}$. hence there must be an infinite set $A_{0} \subseteq A$ of summands occurring in representations of $a^{*} . A_{0}$ must contain an infinite ascending sequence $\left(a_{i}: i<\omega\right)$. But then $\left(a^{*}-a_{i}: i<\omega\right)$ is an infinite descending sequence of elements of $\mathrm{A}^{+}$, contradicting Lemma 1 .

The following lemmas are proved similarly.

LEMMA 3. If $A$ and $B$ are wellordered subsets of a linearly ordered additive abelian group, then each element of $A+B$ has only finitely many representations as $a+b$ where $a \in A$ and $b \in B$.

LEMMA 4. If $A$ and $B$ are wellordered subsets of a linearly ordered additive abelian group, then $A+B$ is wellordered.

A multiplicative version of Lemmas 3 and 4 is

LEMMA 5. Suppose $A$ and $B$ are wellordered subsets of an ordered field, $A, B>$ 0 . Then $A B$ is wellordered and each element of $A B$ has only finitely many representations $a$ s $a b$ where $a \in A$ and $b \in B$.

Now we introduce an essential tool. Suppose $K$ is an ordered field. A control ideal is an ideal $P$ of subsets of $K$ such that

(i) $P$ contains all finite sets,

(ii) if $A, B \in \mathcal{P}$, then $A+B \in \mathcal{P}$,

(iii) if $A \in \mathcal{P}$ and $A>0$, then $A^{+} \in \mathcal{P}$, 
(iv) if $A \in P, c \in K, c>0$ then $c A \in P$,

(v) each $A \in P$ is wellordered.

In order to give an example and for further use we define the following concept.

If $(G,<)$ is a linear ordering, $G_{0} \subseteq G$, then we say that $G_{0}$ is $\mathcal{N}$-like (N) $\mathcal{N}^{*}$-like) in $G$ if for each $a \in G\left\{g \in G_{0}: g<a\right\}\left(\left\{g \in G_{0}: g>a\right\}\right.$ respectively) is finite.

If we define $P_{0}$ to be the set of all $N$-like subsets of the field $\mathbf{R}$ of real numbers with the natural ordering, then $P_{0}$ is a control ideal and the order type $|A|$ of each $A \in P_{0}$ is $\leq \omega$.

Let us fix some ordered field $K$, some control ideal $P$ of subsets of $K$ and some archimedean ordered multiplicative abelian group $G$.

A powerseries in $G$ with coefficients in $K$ is a map $f: G \rightarrow K$ such that $\operatorname{supp}(f):=\{g \in G: f(g) \neq 0\}$ is $\mathcal{N}^{*}$-like in $G . K((G))$ denotes the set of all powerseries in $G$ with coefficients in $K$. For $f_{1}, f_{2} \in K((G)) f_{1}+f_{2} \in K((G))$ is defined by $\left(f_{1}+f_{2}\right)(g)=f_{1}(g)+f_{2}(g) . f_{1} f_{2}$ can be defined by

$$
\left(f_{1} f_{2}\right)(g)=\sum\left\{f_{1}\left(g_{1}\right) f_{2}\left(g_{2}\right): g_{1} \in \operatorname{supp}\left(f_{1}\right), g_{2} \in \operatorname{supp}\left(f_{2}\right) \text { and } g_{1} g_{2}=g\right\} \text {. }
$$

$f \in K((G))$ is said to be positive $(f>0)$ if $\operatorname{supp}(f) \neq \varnothing$ and $f(\max \operatorname{supp}(f))>0$. With these definitions $K((G))$ becomes an ordered field. Moreover in $[\mathbf{F}]$ it is shown that for each sequence $\left(\lambda_{i}: i<\omega\right)$ of elements of $K$ and each $f \in K((G)) \sum_{i=0}^{\infty} \lambda_{i} f^{i}$ makes sense in $K((G))$, provided that $\operatorname{supp}(f)<1$.

For each $f \in K((G)), g_{0} \in G$, the restriction of $f$ to $g_{0}$, denoted by $f ! g_{0}$, is defined by

$$
\left(f ! g_{0}\right)(g)= \begin{cases}f(g) & \text { if } g>g_{0}, \\ 0 & \text { otherwise. }\end{cases}
$$

Now we are ready to define the central concept of our investigation.

Suppose we are given an ordered field $K$, a control ideal $P$ of subsets of $K$ and an archimedean ordered abelian group $G$. A set $A \subseteq K((G))$ is said to be a tree with branch control $P$ if and only if

(a) for each $f \in G, f^{*} \in A$

$$
\left\{f(g): f \in A, f ! g=f^{*} ! g\right\} \in P,
$$

(b) $G_{A}:=\{\max \operatorname{supp}(f): f \in A\}$ is $\mathcal{N}$-like in $G$,

(c) for each $g \in G_{A}$ there is an $\mathcal{N}^{*}$-like set $G_{g} \subseteq G$ such that $\operatorname{supp}(f) \subseteq G_{g}$ for each $f \in A$ such that $\max \operatorname{supp}(f)=g$,

(d) there is some $h \in G$, called a height of $A$, such that for each $f^{*} \in A\{f \in$ $\left.A: f ! h=f^{*} ! h\right\}$ is finite,

(e) $A$ is wellordered.

LEMMA 6 (MAIN LEMMA ON TREES). For each ordered field $K$, each control ideal $P$ of subsets of $K$ and each archimedean ordered abelian group $G$ the set of all trees with branch control $P$ is a control ideal in $K((G))$.

PROOF. It is straightforward to check that the trees with branch control $P$ form an ideal. We have to verify (i)-(v). (i) is obvious. In order to check (ii) let $A, A^{\prime}$ be trees with branch control $P$. Let $G_{A}, G_{A^{\prime}}, G_{g}, G_{g}^{\prime}$ be as in (b), (c) for $A$ and $A^{\prime}$ respectively. We have to prove (a)-(e) for $A+A^{\prime}$. To prove (a) fix some $f^{+} \in A+A^{\prime}$, $g \in G$. Clearly $A ! g$ and $A^{\prime} ! g$ are again wellordered, $\left(A+A^{\prime}\right) ! g=A ! g+A^{\prime} ! g$ and by Lemma $3 f^{+} ! g$ has only finitely many representations $f^{+} ! g=a ! g+a^{\prime} ! g$, where $a ! g \in A ! g$ and $a^{\prime} ! g \in A^{\prime} ! g$. 
Let $S:=\left\{a ! g: a \in A, f^{+} ! g=a ! g+a^{\prime} ! g\right.$ for some $\left.a^{\prime} \in A^{\prime}\right\}$. $S^{\prime}$ is defined similarly. As we have seen, $S$ and $S^{\prime}$ are finite. For each $s \in S \cup S^{\prime}$ let $C_{s}:=\{a(g): a \in$ $A, a ! g=s\}, C_{s}^{\prime}:=\left\{a^{\prime}(g): a^{\prime} \in A^{\prime}, a^{\prime} ! g=s\right\}$. Then $\left\{\left(a+a^{\prime}\right)(g):\left(a+a^{\prime}\right) ! g=\right.$ $\left.f^{+} ! g, a \in A, a^{\prime} \in A^{\prime}\right\}$ is contained in $\bigcup\left\{C_{s}+C_{s^{\prime}}^{\prime}: s \in S, s^{\prime} \in S^{\prime}\right\}$ and this set belongs to $P$. This proves (a).

In order to prove (b) we first claim

$$
\begin{aligned}
G^{+}:=\{ & g \in G_{A} \cup G_{A^{\prime}}: \text { there are } a \in A, a^{\prime} \in A^{\prime} \text { such that } \\
& \left.\max \operatorname{supp}(a)=\max \operatorname{supp}\left(a^{\prime}\right)=g>\max \operatorname{supp}\left(a+a^{\prime}\right)\right\} \\
& \text { is finite. }
\end{aligned}
$$

Assume $G^{+}$is infinite. Then it must contain an infinite ascending chain $g_{1}<$ $g_{2}<\cdots$. Let $a_{i} \in A, a_{i}^{\prime} \in A^{\prime}$ be such that $g_{i}=\max \operatorname{supp}\left(a_{i}\right)=\max \operatorname{supp}\left(a_{i}^{\prime}\right)>$ $\max \operatorname{supp}\left(a_{i}+a_{i}^{\prime}\right)$. Then for all $i a_{i} \ll a_{i+1} \bmod K, a_{i}^{\prime} \ll a_{i+1}^{\prime} \bmod K$. Since $\left(a_{i}+a_{i}^{\prime}\right) \ll g_{i} \bmod K, a_{i}\left(g_{i}\right)=-a_{i}^{\prime}\left(g_{i}\right) \neq 0$. We can assume without loss of generality that $a_{i}\left(g_{i}\right)>0$ for all $i$. But then $\left(a_{i}^{\prime}: i<\omega\right)$ is an infinite descending chain in $A^{\prime}$, contradicting the fact that $A^{\prime}$ is wellordered. This proves claim (1).

In order to prove (b) for $A+A^{\prime}$ it suffices to show that $\left\{\max \operatorname{supp}\left(a+a^{\prime}\right): a \in\right.$ $\left.A, a^{\prime} \in A^{\prime}\right\} \backslash\left(G_{A} \cup G_{A^{\prime}}\right)$ is finite. Assume that there are infinitely many $g_{i}:=$ $\max \operatorname{supp}\left(a_{i}+a_{i}^{\prime}\right)$ outside $G_{A} \cup G_{A^{\prime}}$. Then for each $i$

$$
\max \operatorname{supp}\left(a_{i}\right)=\max \operatorname{supp}\left(a_{i}^{\prime}\right) \in G^{+} .
$$

Because of (1) we can assume that there is some $g^{+} \in G^{+}$such that max $\operatorname{supp}\left(a_{i}\right)=$ $\max \operatorname{supp}\left(a_{i}^{\prime}\right)=g^{+}$for each $i$. Then $\operatorname{supp}\left(a_{i}\right) \subseteq G_{g^{+}}, \operatorname{supp}\left(a_{i}^{\prime}\right) \subseteq G_{g^{+}}^{\prime}$, hence $g_{i} \in G_{g^{+}} \cup G_{g^{+}}^{\prime}$. Since this set is $\mathcal{N}^{*}$-like, we can assume $g_{i+1}<g_{i}<h$ for all $i$, where $h$ is a height for both $A$ and $A^{\prime}$. From Lemma 3 we infer that the set of all $a_{i} ! g_{0}, a_{i}^{\prime} ! g_{0}$ is finite, since for each $i a_{i} ! g_{0}+a_{i}^{\prime} ! g_{0}=0$. But since $g_{i}<h$, there are only finitely many candidates for the $a_{i}, a_{i}^{\prime}$ for fixed values of $a_{i} ! g_{0}, a_{i}^{\prime} ! g_{0}$ respectively, and the finitely many $a_{i}, a_{i}^{\prime}$ can yield only finitely many $g_{i}$-a contradiction proving (b). In order to prove (c) fix some $g^{+} \in G_{A+A^{\prime}}$.

$$
G_{g^{+}}^{+}:=\bigcup\left\{\operatorname{supp}\left(a+a^{\prime}\right): a \in A, a^{\prime} \in A^{\prime}, g^{+}=\max \operatorname{supp}\left(a+a^{\prime}\right)\right\} .
$$

We consider some $a \in A, a^{\prime} \in A^{\prime}$ such that $g^{+}=\max \operatorname{supp}\left(a+a^{\prime}\right)$ and distinguish two cases.

(1) $g^{+}<\max \operatorname{supp}(a)=\max \operatorname{supp}\left(a^{\prime}\right)$. In this case

$$
\max \operatorname{supp}(a)=\max \operatorname{supp}\left(a^{\prime}\right) \in G^{+} .
$$

(2) $g^{+}=\max \left(\max \operatorname{supp}(a), \max \operatorname{supp}\left(a^{\prime}\right)\right)$, say

$$
g^{+}=\max \operatorname{supp}(a) \geq \max \operatorname{supp}\left(a^{\prime}\right) .
$$

In this case

$$
\max \operatorname{supp}\left(a^{\prime}\right) \in\left\{g \in G_{A^{\prime}}: g \leq g^{+}\right\} .
$$

In each case we have

$\max \operatorname{supp}(a), \max \operatorname{supp}\left(a^{\prime}\right) \in G^{+} \cup\left\{g \in G_{A}: g \leq g^{+}\right\} \cup\left\{g \in G_{A^{\prime}}: g \leq g^{+}\right\}$. 
Let $G^{++}$denote this set. $G^{++}$is finite. Now clearly

$$
\begin{aligned}
G_{g^{+}}^{+} & \subseteq \bigcup\left\{\operatorname{supp}(a) \cup \operatorname{supp}\left(a^{\prime}\right): a \in A, a^{\prime} \in A^{\prime},\right. \\
& \left.\max \operatorname{supp}(a) \in G^{++} \text {and } \max \operatorname{supp}\left(a^{\prime}\right) \in G^{++}\right\} \\
& \subseteq \bigcup\left(\left\{G_{g}: g \in G^{++} \cap G_{A}\right\} \cup\left\{G_{g}^{\prime}: g \in G^{++} \cap G_{A^{\prime}}\right\}\right)
\end{aligned}
$$

and this set is $\mathcal{N}^{*}$-like in $G$. Thus we have proved (c).

Suppose $h$ is a height of both $A$ and $A^{\prime}$. By Lemma 3 for each $a^{+} \in A+A^{\prime}$ there are only finitely many $a ! h, a^{\prime} ! h$ such that $\left(a+a^{\prime}\right) ! h=a^{+} ! h, a \in A, a^{\prime} \in A^{\prime}$. Hence $h$ is also a height of $A+A^{\prime}$.

(e) is just a special case of Lemma 4 . Thus we have shown that $A+A^{\prime}$ is a tree.

In order to verify (iii) assume that $A$ is a tree with branch control $P$ and $A>0$. Fix some $a^{*} \in A^{+}, g \in G$. Let $S$ be the finite set of nonzero summands occurring in representations of $a^{*} ! g$ as a sum of elements of $A ! g$ and let $k$ be an upper bound for the lengths of these sums. For each $s \in S$ put

$$
C_{s}:=\{a(g): a \in A, a ! g=s\} .
$$

Then $C_{s} \in \mathcal{P}$. Now if $c \in\left\{a(g): a \in A^{+}, a ! g=a^{*} ! g\right\}$, then there are an $m \leq k$ and $s_{1}, \ldots, s_{m} \in S, a_{1}, \ldots, a_{m+n} \in A$ such that $s_{1}+\cdots+s_{m}=a^{*} ! g, a_{1} ! g=$ $s_{1}, \ldots, a_{m} ! g=s_{m}, a_{m+1} ! g=\cdots=a_{m+n} ! g=0$, and $c=a_{1}(g)+\cdots+a_{m+n}(g)$. Now $a_{1}(g) \in C_{s_{1}}, \ldots, a_{m}(g) \in C_{s_{m}}$ and $a_{m+1}(g), \ldots, a_{m+n}(g) \in\{a(g): a \in A, a ! g=0\}$. Let

$$
C=\{a(g): a \in A, a ! g=0, a(g) \neq 0\} .
$$

Clearly $C \in \mathcal{P}$ and $C>0$. Since $a_{m+1}(g)+\cdots+a_{m+n}(g) \in C^{+} \in \mathcal{P}, c \in$ $\bigcup\left\{C_{s_{1}}+\cdots+C_{s_{m}}+C^{+}: m \leq k, s_{1}, \ldots, s_{m} \in S\right\} \in \mathcal{P}$. Since $c$ was an arbitrary element of $\left\{a(g): a \in A^{+}, a ! g=a^{*} ! g\right\}$, this set also belongs to $\mathcal{P}$ and we have verified (a) for $A^{+}$.

Put $G_{A}:=\{\max \operatorname{supp}(a): a \in A\}$. Since each element of $A$ is positive, for all $a_{1}, \ldots, a_{n} \in A \max \operatorname{supp}\left(a_{1}+\cdots+a_{n}\right) \in G_{A}$ and we have $G_{A^{+}}=G_{A}$. We fix some $g^{+} \in G_{A^{+}}$. If $g^{+}=\max \operatorname{supp}\left(a_{1}+\cdots+a_{n}\right)$, then $\max \operatorname{supp}\left(a_{1}\right), \ldots, \max \operatorname{supp}\left(a_{n}\right)$ $\leq g^{+}$. Therefore $\operatorname{supp}\left(a_{1}+\cdots+a_{n}\right) \subseteq \bigcup\left\{G_{g}: g \in G_{A}, g \leq g^{+}\right\}$and this is a finite union of $\mathcal{N}^{*}$-like sets, hence $\mathcal{N}^{*}$-like. Thus we have proved (b) and (c).

Let $h_{0}$ bè a height of $A$. Then $\left\{a \in A: a ! h_{0}=0\right\}$ is finite. Choose $h<$ $\min \left(\left\{h_{0}\right\} \cup\left\{\max \operatorname{supp}(a): a \in A, a ! h_{0}=0\right\}\right)$. Then $A ! h>0$. Let us fix some $a^{+} \in A^{+}$. Since there are only finitely many representations of $a^{+} ! h$ as a sum of elements of $A ! h$ and since $h$ is a height of $A,\left\{a \in A^{+}: a ! h=a^{+} ! h\right\}$ is finite, i.e., $h$ is a height of $A^{+} . A^{+}$is wellordered by Lemma 1 , hence $A^{+}$is a tree.

Now let $A$ be an arbitrary tree with branch control $P$ and let $a_{0}$ be a positive element of $K((G))$. Let $g_{0}$ denote $\max \operatorname{supp}\left(a_{0}\right)$ and $c_{0}:=a_{0}\left(g_{0}\right)$. Then $a_{0}=$ $c_{0} g_{0}\left(1+r_{0}\right)$ where $r_{0} \in K((G))$ is such that $\operatorname{supp}\left(r_{0}\right)<1$. It is easy to see that for each tree $B$ with branch control $P c_{0} B$ and $g_{0} B$ are again such trees. Therefore it suffices to check (a)-(e) for $\left(1+r_{0}\right) A$. Fix $a^{*} \in A, g \in G$. We have to show that

$$
C:=\left\{\left(\left(1+r_{0}\right) a\right)(g): a \in A,\left(\left(1+r_{0}\right) a\right) ! g=\left(\left(1+r_{0}\right) a^{*}\right) ! g\right\} \in \mathcal{P} .
$$

Observe that, for arbitrary $a_{1}, a_{2}, a_{1} ! g=a_{2} ! g$ iff $a_{1}-a_{2}=c g+o_{K}(g)$ for some $c \in K$. Therefore

$$
a_{1} ! g=a_{2} ! g \quad \text { iff } a_{1}\left(1+r_{0}\right) ! g=a_{2}\left(1+r_{0}\right) ! g .
$$


Hence $C=\left\{\sum\left\{\left(1+r_{0}\right)\left(g_{1}\right) a\left(g_{2}\right): g_{1} g_{2}=g\right\}: a \in A, a ! g=a^{*} ! g\right\}$,

$$
C=\left\{a(g)+\sum\left\{r_{0}\left(g_{1}\right) a^{*}\left(g_{2}\right): g_{1} g_{2}=g, g_{1}<1, g_{2}>g\right\}: a \in A, a ! g=a^{*} ! g\right\} .
$$

Put $d:=\sum\left\{r_{0}\left(g_{1}\right) a^{*}\left(g_{2}\right): g_{1} g_{2}=g, g_{1}<1, g_{2}>g\right\}$. Then $C=\{d\}+\{a(g): a \in$ $\left.A, a ! g=a^{*} ! g\right\} \in \mathcal{P}$. Clearly $\left\{\max \operatorname{supp}\left(\left(1+r_{0}\right) a\right): a \in A\right\}=\{\max \operatorname{supp}(a): a \in$ $A\}$, hence (b) is obvious.

For each $g \in G, a \in A$ such that $\max \operatorname{supp}\left(\left(1+r_{0}\right) a\right)=g$ we have

$$
\operatorname{supp}\left(\left(1+r_{0}\right) a\right) \subseteq\left(\operatorname{supp}\left(r_{0}\right)\right) G_{g} .
$$

This set is a product of $\mathcal{N}^{*}$-like sets, hence $\mathcal{N}^{*}$-like.

Because of (2), each height of $A$ is also a height of $\left(1+r_{0}\right) A$. Condition (e) is also obvious. This completes the proof of (iv). Finally, (v) for trees coincides with (e).

Our next task is to examine some basic operations with trees.

Lemma 7 (Horizontal MUlTipliCation Lemma). Let $C \in P$ consist of positive elements and let for each $c \in C A_{c}$ be a tree with branch control $P$ such that $A_{c} \sim 1 \bmod K$. Assume moreover that there is a common height $h<1$ for all the trees $A_{c}$ and $\operatorname{supp}\left(A_{c}\right) \subseteq G_{0}$ for some $\mathcal{N}^{*}$-like set $G_{0}$ and for each $c \in C$.

Then $\bigcup\left\{c A_{c}: c \in C\right\}$ is again a tree with branch control $P$ and height $h$.

We leave the easy proof to the reader.

Lemma 8 (Vertical Multiplication Lemma), Let $G_{0} \subseteq G$ be $\mathcal{N}$-like. For each $g \in G_{0}$ let $A_{g}$ be a tree with branch control $P$ such that $\max \operatorname{supp}\left(A_{g}\right)=$ $\{1\}$ and $A_{g}>0$. Suppose moreover that there is a common height for all trees $A_{g}$. Then $\bigcup\left\{g A_{g}: g \in G_{0}\right\}$ is again a tree with branch control $P$.

PROOF. The only point which is not obvious for $\bigcup\left\{g A_{g}: g \in G_{0}\right\}$ is (a). Therefore let us fix some $g^{*} \in G_{0}$ and some $a^{*} \in A_{g^{*}}, g_{0} \in G$. We distinguish two cases.

(1) $\left(g^{*} A^{*}\right) ! g_{0}=0$.

If $(g a) ! g_{0}=0$ and $(g a)\left(g_{0}\right) \neq 0$, then $g_{0}=\max \operatorname{supp}(g a)=g$. Therefore $\left\{(g a)\left(g_{0}\right): g \in G_{0}, a \in A_{g},(g a) ! g_{0}=0\right\}$ is a subset of

$$
\left\{\left(g_{0} a\right)\left(g_{0}\right): a \in A_{g_{0}}\right\} \cup\{0\}=\{0\} \cup\left\{a(1): a \in A_{g_{0}}\right\} \in \mathcal{P} .
$$

(2) $\left(g^{*} a^{*}\right) ! g_{0} \neq 0$, i.e. $g^{*}=\max \operatorname{supp}\left(g^{*} a^{*}\right)>g_{0}$.

Then if $(g a) ! g_{0}=\left(g^{*} a^{*}\right) ! g_{0}$, then $g=\max \operatorname{supp}(g a)=g^{*}$. Therefore

$$
\begin{aligned}
& \left\{(g a)\left(g_{0}\right): g \in G_{0}, a \in A_{g},(g a) ! g_{0}=\left(g^{*} a^{*}\right) ! g_{0}\right\} \\
& \quad=\left\{a\left(g_{0}\left(g^{*}\right)^{-1}\right): a \in A_{g^{*}}, a !\left(g_{0}\left(g^{*}\right)^{-1}\right)=a^{*} !\left(g_{0}\left(g^{*}\right)^{-1}\right)\right\} \in \mathcal{P} .
\end{aligned}
$$

LEMMA 9 (LEMMA ON ORDER TYPES). If $P$ is a control ideal such that $|C| \leq \alpha$ for each $C \in P$, where $\alpha$ is an infinite ordinal, then each tree with branch control $P$ has an order type $\leq \alpha^{\omega}$.

Proof. Let $A$ be a tree with branch control $P$, let $G_{A}$ and $G_{g}$ be as in (b), (c), and let $h$ be a height of $A$. For each $g_{0} \in G_{A}$ we put $A_{g_{0}}:=\{a \in A: \max \operatorname{supp}(a)=$ $\left.g_{0}\right\}$. Then $\operatorname{supp}\left(A_{g_{0}}\right) \subseteq G_{g_{0}}$. Let $\left\{g \in G_{g_{0}}: g>h\right\}=\left\{g_{1}, \ldots, g_{n}\right\}$ where $g_{1}>$ $\cdots>g_{n}$. Then each $a ! h \in A_{g_{0}} ! h$ is uniquely characterized by its coefficients 
$a\left(g_{1}\right), \ldots, a\left(g_{n}\right)$. Hence $A_{g_{0}} ! h$ can be viewed as the lexicographic ordering of some $n$-letter words, where each letter is chosen from a set of order type $\leq \alpha$. Therefore $\left|A_{g_{0}} ! h\right| \leq \alpha^{n}<\alpha^{\omega}$. Since $A_{g_{0}}$ is obtained from $A_{g_{0}} ! h$ by replacing each $a_{0} ! h$ by the finite set $\left\{a \in A_{g_{0}}: a ! h=a_{0} ! h\right\}$, also $\left|A_{g_{0}}\right|<\alpha^{\omega}$. let $A_{g_{0}}^{\prime}, A_{g_{0}}^{\prime \prime}$ denote the set of positive, respectively negative, elements of $A_{g_{0}}$. Since $A$ is wellordered, $A_{g_{0}}^{\prime \prime}=\varnothing$ for almost all $g_{0} \in G_{A}$. Let $G_{A} \subseteq\left\{g_{i}: i<\omega\right\}$, where $g_{0}<g_{1}<\cdots$. Assume $A_{g_{i}}^{\prime \prime}=\varnothing$ for each $i>n$. Then

$$
|A| \leq \sup \left\{\left|A_{g_{n-1}}^{\prime \prime}\right|+\cdots+\left|A_{g_{0}}^{\prime \prime}\right|+1+\left|A_{g_{0}}^{\prime}\right|+\cdots+\left|A_{g_{k}}^{\prime}\right|: k \in \omega\right\} .
$$

Since $\alpha^{\omega}$ is closed under addition and $\left|A_{g_{i}}^{\prime}\right|,\left|A_{g_{i}}^{\prime \prime}\right| \leq\left|A_{g_{i}}\right|$, we obtain $|A| \leq \alpha^{\omega}$.

LEMMA 10. Suppose $\lambda_{i} \in K$ for each $i \in \omega, \lambda_{1}>0$, and for each $a \in K((G))$ with $\operatorname{supp}(a)<1, \Phi(a)$ is defined by

$$
\Phi(a)=\sum_{i=0}^{\infty} \lambda_{i} a^{i} .
$$

Let $A$ be a tree with branch control $P$ such that $\operatorname{supp}(A)<1$ and $\operatorname{supp}(A)$ is $\mathcal{N}^{*}$-like.

Then $\Phi(A)$ is also a tree with branch control $P$ and each height of $A$ is also a height of $\Phi(A)$.

ProOF. We show first that for all $a, b \in K((G))$ such that $\operatorname{supp}(a)<1$, $\operatorname{supp}(b)<1$ and for all $g \in G$

$$
a ! g=b ! g \quad \text { iff } \Phi(a) ! g=\Phi(b) ! g .
$$

For $g \geq 1$ this is obvious. We assume $g<1$. Since $G$ is archimedean ordered, there is some $n$ such that $b^{i}, a^{i} \ll g \bmod K$ for each $i>n$. Therefore $\Phi(a)=$ $\sum_{i=0}^{n} \lambda_{i} a^{i}+o_{K}(g), \Phi(b)=\sum_{i=0}^{n} \lambda_{i} b^{i}+o_{K}(g)$. Now, if $b=a+c g+o_{K}(g)$ for some $c \in K$, then for each $i \geq 2 b^{i}=a^{i}+o_{K}(g)$. Hence

$$
\Phi(b)=\Phi(a)+\lambda_{1} c g+o_{K}(g) \text {. }
$$

Since the assumption is fulfilled if $b ! g=a ! g, b ! g=a ! g$ implies $\Phi(b) ! g=\Phi(a) ! g$. If $b ! g \neq a ! g$, then $g^{\prime}:=\max \operatorname{supp}(b-a)>g, b=a+c g^{\prime}+o_{K}\left(g^{\prime}\right)$ for some $c \in K$, $c \neq 0$, hence $\Phi(b)-\Phi(a) \sim \lambda_{1} c g^{\prime} \bmod K$, i.e. $\max \operatorname{supp}(\Phi(b)-\Phi(a))=g^{\prime}>g$ which means $\Phi(b) ! g \neq \Phi(a) ! g$. This proves (3).

We have to check (a)-(e) for $\Phi(A)$.

Let us fix some $g \in G$. We observe that for each $a \in K((G))$ with $\operatorname{supp}(a)<1$ and for each $i \geq 2$,

$$
a^{i}(g)=\sum\left\{a\left(g_{1}\right) a\left(g_{2}\right) \cdots a\left(g_{i}\right): g_{1} g_{2} \cdots g_{i}=g\right\}
$$

depends only on $a ! g$. Moreover, since $g$ has only finitely many representations as $g_{1} g_{2} \cdots g_{n}, a^{i}(g)=0$ for almost every $i$. We can denote $\sum_{i=1}^{\infty} \lambda_{i} a^{i}(g)$ by $r(a ! g)$. Then for each $a^{*} \in A$

$$
\begin{aligned}
\{\Phi(a)(g): & \left.a \in A, \Phi(a) ! g=\Phi\left(a^{*}\right) ! g\right\}
\end{aligned}
$$

Since $P$ satisfies (i), (ii), and (iv), this set belongs to $P$ and we have proved (a). 
If $\lambda_{0} \neq 0$, then $\{\max \operatorname{supp}(\phi(a)): a \in A\}=\{1\}$, otherwise $\{\max \operatorname{supp}(\Phi(a))$ : $a \in A\}=G_{A}$. In each case this set is finite. Clearly, each element of $\operatorname{supp}(\Phi(A))$ is 1 or a finite product of elements of $\operatorname{supp}(A)$. Hence $\operatorname{supp}(\Phi(A))$ is $\mathcal{N}^{*}$-like too. If $h$ is a height of $A$, then it is also a height of $\Phi(A)$ because of (3).

If $a<b$, say $b=a+c g+o_{K}(g)$ for some $c \in K, c>0$, then by (4) $\Phi(b)=$ $\Phi(a)+\lambda_{1} c g+o_{K}(g)>\Phi(a)$, hence $\Phi$ is strictly monotonic increasing and $\Phi(A)$ inherits the wellordering of $A$.

Now we are ready to apply our concepts.

Let $R$ also denote the ordered multiplicative group of all positive real numbers. For each $i \in \omega$, let $G_{2 i+1}:=x^{x^{i \mathbf{z}}}, G_{2 i}:=R^{x^{i \mathbf{z}}}, K_{0}:=\mathbf{R}, K_{i+1}:=K_{i}\left(\left(G_{i}\right)\right)$. We have already defined $P_{0}$ to be the control ideal of all $\mathcal{N}$-like sets of real numbers. $P_{i+1}$ denotes the set of all subsets of $K_{i+1}$ which are trees with branch control $P_{i}$. By the Main Lemma on Trees $P_{i+1}$ is a control ideal.

As a direct application of the last lemma we prove

Lemma 11. For each $i \in \omega, A \in \mathcal{P}_{i+1}$ such that $A \sim 1 \bmod K_{i}$, the set $A^{x}$ belongs also to $P_{i+1}$ and each height of $A$ is a height of $A^{x}$.

Proof. Let $A_{0}:=A-1$. Then $A_{0} \in \mathcal{P}_{i+1}$ and $\operatorname{supp}\left(A_{0}\right)<1$. Applying Lemma 10 we see that $\ln (A)=\ln \left(1+A_{0}\right) \in \mathcal{P}_{i+1}$ and heights of $A$ are also heights of $\ln (A)$. From the form of the $\ln$-series it is clear that $\operatorname{supp}(\ln (A))<1$, hence $\operatorname{supp}(\ln (A)) \leq(1 / x) . x \ln (A)$ is also a tree with branch control $P_{i}$.

If $i>0$, then $x \in K_{i}$ and $\operatorname{supp}(x \ln (A))<1$. In this case by Lemma 10 $A^{x}=\exp (x \ln (A)) \in \mathcal{P}_{i+1}$ and each height of $A$ is also a height of $A^{x}$.

If $i=0, \operatorname{supp}(x \ln (A)) \leq 1$. Putting $C:=\{a(1): a \in x \ln (A)\} \in \mathcal{P}_{0}, A_{c}:=$ $\{a-c: a \in x \ln (A), a(1)=c\}$ for each $c \in C$ we obtain trees $A_{c}$ with $\operatorname{supp}\left(A_{c}\right)<1$. Heights of $A$ are also heights of the $A_{c}$. Now $A^{x}=\bigcup\left\{e^{c} \exp \left(A_{c}\right): c \in C\right\} \in \mathcal{P}_{1}$ by the Horizontal Multiplication Lemma and Lemma 10.

If $K, L$ are ordered fields, $K \subseteq L$ and $a \in L$, then we say that $a$ is $K$-separated if there is some positive $\varepsilon \in K$ such that $\varepsilon<|a|<\varepsilon^{-1}$. Following our conventions, $A \subseteq L$ is $K$-separated if each $a \in A$ has this property.

Lemma 12. For each $i \in \omega, A \in \mathcal{P}_{i+2}$ such that $A$ is $K_{i}$-separated and $A>0$, $x^{\mathbf{N}} \cdot A^{x} \in \mathcal{P}_{i+2}$.

Proof. We prove the assertion by induction on $i$. If $i=0$, then $A$ is $\mathbf{R}$ separated. Since $A \in \mathcal{P}_{2}, A^{\prime}:=\{f(1): f \in A\}$ belongs to $P_{1}$ and is $\mathbf{R}$-separated too. for each $a \in A^{\prime}$ we put $A_{a}:=(1 / a)\{f \in A: f(1)=a\}$. Then $A=\bigcup\left\{a A_{a}: a \in A^{\prime}\right\}$. If $h$ is a height of $A$, then it is a height of each $A_{a}$. Let $h^{\prime}$ be a height of $A^{\prime} . C:=$ $\left\{a(1): a \in A^{\prime}\right\}$ is $\mathcal{N}$-like, $C>0$. For each $c \in C$ let $A_{c}^{\prime}:=(1 / c)\left\{a \in A^{\prime}: a(1)=c\right\}$. Then $A_{c}^{\prime} \sim 1 \bmod K_{0}, A_{c}^{\prime} \in \mathcal{P}_{1}$ and $A^{\prime}=\bigcup\left\{c A_{c}^{\prime}: c \in C\right\}$. Now

$$
x^{\mathbf{N}} \cdot A^{x}=\bigcup\left\{c^{x} x^{\mathbf{N}} d^{x} A_{c d}^{x}: c \in C, d \in A_{c}^{\prime}\right\} .
$$

By Lemma $11 A_{c}^{\prime x} \in \mathcal{P}_{1}$ and this set has height $h^{\prime}$. It is easy to see that $A_{c}^{\prime x}$ is $K_{0}$-separated. By the Vertical Multiplication Lemma $B_{c}^{\prime}:=x^{\mathbf{N}} \cdot A_{c}^{\prime x} \in \mathcal{P}_{1}$ for each $c \in C$. Each $a \in B_{c}^{\prime}$ has the form $x^{n} d^{x}$ for uniquely determined $n \in \omega, d \in A_{c}^{\prime}$-we put $B_{a}:=A_{c d}^{x}$. Because of (5)

$$
x^{\mathbf{N}} \cdot A^{x}=\bigcup\left\{c^{x} a B_{a}: c \in C, a \in B_{c}^{\prime}\right\} .
$$


By Lemma $11, B_{a} \in P_{2}$ and each $B_{a}$ has the height $h$.

Since $x \in K_{1}$ and $A_{c d} \sim 1 \bmod K_{1}$ for all $c, d$, also $B_{a} \sim 1 \bmod K_{1}$. The Horizontal Multiplication Lemma can be applied to see that for each $c \in C B_{c}^{*}:=$ $\bigcup\left\{a B_{a}: a \in B_{c}^{\prime}\right\} \in P_{2}$ and $B_{c}^{*}$ has height $h$. Since $B_{a} \sim 1 \bmod K_{1}, B_{c}^{\prime} \subseteq K_{1}$, $B_{c}^{\prime}>0, B_{c}^{*}$ is $K_{1}$-separated. Now by (6) and by the Vertical Multiplication Lemma

$$
x^{\mathbf{N}} \cdot A^{x}=\bigcup\left\{c^{x} B_{c}^{*}: c \in C\right\} \in \mathcal{P}_{2} .
$$

Now assume $i>0$ is such that the assertion has been proved for $i-1$, let $A \in P_{i+2}$ be $K_{i}$-separated. $A^{\prime}:=\{f(1): f \in A\} \in \mathcal{P}_{i+1}$ is also $K_{i}$-separated and $A^{\prime}>0$. For each $a \in A^{\prime}$ we put

$$
A_{a}:=(1 / a)\{f \in A: f(1)=a\} .
$$

$A_{a} \sim 1 \bmod K_{i+1} . A_{a}^{x} \in \mathcal{P}_{i+2}$ by Lemma 11. Moreover $A=\bigcup\left\{a A_{a}: a \in A^{\prime}\right\}$. If $h$ is a height of $A$, then it is a height of each $A_{a}^{x}$ and $A_{a}^{x} \sim 1 \bmod K_{i+1}$. Let $h^{\prime}$ be a height of $A^{\prime}$ and let $C:=\left\{\max \operatorname{supp}(a(1)): a \in A^{\prime}\right\}$.

Since $\left\{a(1): a \in A^{\prime}\right\} \in \mathcal{P}_{1}, C$ is an $\mathcal{N}$-like subset of $G_{i}$. For each $c \in C$ put $A_{c}^{\prime}:=(1 / c)\left\{a \in A^{\prime}: c=\max \operatorname{supp}(a(1))\right\}$. Then $A_{c}^{\prime} \in \mathcal{P}_{i+1} . h^{\prime}$ is also a height of each $A_{c}^{\prime}$. For each $a \in A^{\prime} a(1) \in K_{i}$ and $a / \max \operatorname{supp}(a(1))$ is $K_{i-1}$-separated. Thus $A_{c}^{\prime}$ is $K_{i-1}$-separated and we can apply the induction hypothesis to $A_{c}^{\prime}$, obtaining $B_{c}^{\prime}:=x^{\mathbf{N}} \cdot A_{c}^{\prime x} \in P_{i+1}$. Each $a \in B_{c}^{\prime}$ has the form $x^{n} d^{x}$ where $d \in A_{c}^{\prime}$. By Lemma 5 there are only finitely many possible $d$ 's in such a representation of $a$. Let $B_{a}$ be the union of all the $A_{c d}^{x}$ where $d$ occurs in such a representation of $a$. Each $B_{a}$ has height $h$ and each of its elements has a support consisting of products of elements of $\operatorname{supp}(A)$, forming an $\mathcal{N}^{*}$-like subset of $G_{i+2} \cdot x^{\mathbf{N}} \cdot A^{x}=\bigcup\left\{c^{x} a B_{a}: a \in B_{c}^{\prime}, c \in C\right\}$. $B_{c}^{*}:=\bigcup\left\{a B_{a}: a \in B_{c}^{\prime}\right\} \in \mathcal{P}_{i+2}$ by the Horizontal Multiplication Lemma and has height $h .\left\{c^{x}: c \in C\right\}$ is an $\mathcal{N}$-like subset of $G_{i+2}$. Since $A_{a}^{x} \sim 1 \bmod K_{i+1}$ and $B_{c}^{\prime} \subseteq K_{i+1}$, each $B_{c}^{*}$ is $K_{i+1}$-separated. Now by the Vertical Multiplication Lemma $x^{\mathbf{N}} \cdot A^{x}=\bigcup\left\{c^{x} B_{c}^{*}: c \in C\right\} \in \mathcal{P}_{i+2}$.

Lemma 13. If $A \in \mathcal{P}_{i}$ and $A>0$, then $x^{\mathbf{N}} \cdot A^{x} \in \mathcal{P}_{i+2}$.

PROOF. First we claim:

If $G_{0}$ is an $\mathcal{N}$-like subset of $G_{i+1}$ and for each $g \in G_{0}, C_{g} \in \mathcal{P}_{i}, C_{g}>0$, then $\bigcup\left\{g C_{g}: g \in G_{0}\right\} \in \mathcal{P}_{i+1}$.

Since each $C_{g}$ can be considered as a tree with branch control $P_{i}$ and $\operatorname{supp}\left(C_{g}\right)=$ $\{1\}$, the claim is just a special case of the Vertical Multiplication Lemma.

Case 1: $i=0$. Then $A$ is an $\mathcal{N}$-like subset of $\mathbf{R}$ and $A^{x}$ is an $\mathcal{N}$-like subset of $G_{2}$. Since $x^{\mathbf{N}} \in \mathcal{P}_{1}$ the assertion follows from the claim.

Case 2: $i>0$. Assume $A \in \mathcal{P}_{i}, A>0$. Put $G_{0}:=\{\max \operatorname{supp}(a): a \in A\} . G_{0}^{x}$ is an $\mathcal{N}$-like subset of $G_{i+2}$. For each $g \in G_{0}$ let

$$
A_{g}:=g^{-1}\{a \in A: g=\max \operatorname{supp}(a)\} \text {. }
$$

Then $A=\bigcup\left\{g A_{g}: g \in G_{0}\right\}$ and $A_{g} \in \mathcal{P}_{i}$ is $K_{i-1}$-separated. $A_{g}$ can be viewed as a tree with branch control $P_{i}$. Then we can apply Lemma 12 to see that $x^{\mathbf{N}} \cdot A_{g}^{x} \in \mathcal{P}_{i+1}$. Now the assertion follows by our claim.

THEOREM A. $\operatorname{Sk}\left(2^{x^{i}}\right) \in P_{2 i-1}$ for each $i \geq 1$.

ProOF. Obviously $\operatorname{Sk}\left(2^{x}\right) \in P_{1}$. By a result of Levitz $[\mathbf{L}]$

$$
\operatorname{Sk}\left(2^{x^{i+1}}\right)=\left(x^{\mathbf{N}} \operatorname{Sk}\left(2^{x^{i}}\right)^{x}\right)^{+} .
$$

Hence the result can be proved by induction on $i$ using Lemma 13 and (iii). 
COROLlARY A. $\left|\operatorname{Sk}\left(2^{x^{n}}\right)\right|=\omega^{\omega^{2 n-1}}$.

PROOF. In [DL] there is given a subset of $\operatorname{Sk}\left(2^{x^{n}}\right)$ which has the order type $\omega^{\omega^{2 n-1}}$. On the other hand an induction on $n$ using the Lemma on Order Types shows that $|A| \leq \omega^{\omega^{n}}$ for each $A \in \mathcal{P}_{n}$.

LEMMA 14. $\mathrm{Sk}\left(x^{x^{i+1}}\right)=\left(x^{\mathbf{N}} \mathrm{Sk}\left(x^{x^{i}}\right)^{x}\right)^{+}$and $\mathrm{Sk}\left(x^{x}\right)$ is the set of all functions of the form $\sum_{i \leq n} i^{x} f_{i}$ where $f \in \operatorname{Sk}\left(2^{x}\right) \cup\{0\}$.

PROOF. If $f^{g}<x^{x}$ and $g>\mathbf{N}$, then $g<x^{2}$. Hence $g=c x+d$ for some natural numbers $c, d, c>0$, and $f^{g}=\left(f^{c}\right)^{x} f^{d}$. Also $\left(f^{c}\right)^{x}<x^{x}$, i.e. $f$ must be a natural number. Therefore $f^{g}$ has the form $i^{x} h$ for natural numbers $i, h$, and each integral exponential function below $x^{x}$ must be of the desired form.

Similarly, if $f<x^{x^{i+1}}$, then each subterm of $f$ of the form $g^{h}$ with $g>1$ is such that $h<2^{x}$, i.e. $h$ is a polynomial with natural numbers as coefficients. Hence $g^{h}$ can be represented as $g_{1}^{x} g^{n}$ with a certain term $g_{1}$ and a natural number $n$. But then $g_{1}<x^{x^{i}}$. Moreover, if $h$ has positive degree, then $g \leq g_{1}$. Otherwise $g^{h}$ can be decomposed as a product. Hence each multiplicatively and additively indecomposable function below $x^{x^{i+1}}$ is of the form $1, x$ or $g^{x}$ where $g<x^{x^{i}}$, and the assertion follows.

THEOREM B. $\operatorname{Sk}\left(x^{x^{i}}\right) \in P_{2 i}$.

PROOF. The proof is by induction on $i$ using Lemmas 13 and 14 and (iii).

COROllary B. $\left|\operatorname{Sk}\left(x^{x^{n}}\right)\right|=\omega^{\omega^{2 n}}$.

PROOF. For $n=1$ this is clear. By induction it follows as in the proof of Corollary A that $\left|\operatorname{Sk}\left(x^{x^{n}}\right)\right| \leq \omega^{\omega^{2 n}}$. Since $\left|\operatorname{Sk}\left(2^{x^{n}}\right)\right|=\omega^{\omega^{2 n-1}}$, the order type of the set of all functions of the form $\sum_{i \leq k} 2^{i x^{n}} f_{i}$, where $f_{i}<2^{x^{n}}$, is $\omega^{\omega^{2 n}}$. Hence also $\left|\mathrm{Sk}\left(x^{x^{n}}\right)\right| \geq \omega^{\omega^{2 n}}$.

\section{REFERENCES}

[D] B. I. Dahn, The limit behaviour of exponential terms, Fund. Math. 74 (1984), 169-186.

[DG] B. I. Dahn and P. Göring, Notes on exponential-logarithmic terms, Proc. 2nd Easter Conference on Model Theory, Seminarber. No. 60, Humboldt University, pp. 11-19.

[DL] L. van den Dries and H. Levitz, On Skolem's exponential functions below $2^{2^{x}}$, Trans. Amer. Math. Soc. 286 (1984), 339-349.

[E] A. Ehrenfeucht, Polynomial functions with exponentiation are well ordered, Algebra Universalis 3 (1973), 261-262.

[F] L. Fuchs, Partially ordered algebraic systems, Pergamon Press, Oxford, 1963.

[H] G. H. Hardy, Orders of infinity, 2nd ed., Cambridge Univ. Press, Cambridge, 1924.

[L] H. Levitz, An initial segment of the set of polynomial functions with exponentiation, Algebra Universalis 7 (1977), 133-136.

[Sk] T. Skolem, An ordered set of arithmetic functions representing the least $\varepsilon$-number, Norske Vid. Selsk. Forh. 29 (1956), 54-59.

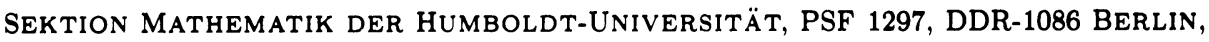
GERMAN DEMOCRATIC REPUBLIC 